\title{
Editor's Note: Special Section on Scalable Data Management
}

Distributed and Parallel Databases gratefully acknowledges the editorial work of the following scholars:

- Shrideep Pallickara, Colorado State University

- Samee Khan, North Dakota State University

- Rodrigo Calheiros, University of Melbourne

- Rajkumar Buyya, University of Melbourne

- Rui Zhang, University of Melbourne

on this special section "Scalable Data Management."

The two papers in this section include:

- "Efficient integrity verification of replicated data in cloud using homomorphic encryption” by Raghul Mukundan, Sanjay Madria, Mark Linderman and

- "OATS: online aggregation with two-level sharing strategy in cloud" by Yuxiang Wang, Junzhou Luo, Aibo Song, Fang Dong 